disc degeneration. When disc degeneration proceeds further, slipping of the vertebrae could be taken place, and patients start to have complaints due to instability toward the final stage of the disc degeneration, the vertebral column loses its mobility. If the degeneration extends to several discs, the vertebral colmn will similate a log.

The development of disc lesion is studied and presented with the aid of $16 \mathrm{~mm}$ cine-discography.

\title{
90. A Study of Pain Pattern Introduced by Arthrography of the Facet Articulation
}

\author{
Tadaatsu Ito, Takashi Nakagawa and Susumu AnazaWA \\ Dept. of Orthopaedic Surgery, Nippon Medical College
}

A study was made of a series of 22 patients with low back pain in terms of elucidation of the pain mechanism by means of injection of hypertonic saline into the facet articulation of the lumbar spine, associated with arthrography. The results obtained from the study were summarized as follows: The patients consist of 10 cases with disc herniation, 9 of spondylolysis and 3 of low back pain of postural origin.

Under careful fluoloscopy, $1.5 \mathrm{cc}$ of $76 \%$ pyraceton with $6 \%$ saline was injected into the facet articulation.

1) The referred pain patterns revealed that from the lumber $4 / 5$ as well as lumbar 5 and sacral 1 the pain referred to gluteal and posterior aspect of the thigh, from the lumbar $1 / 2$ referred to lower abdominal quadrant of the ipsilateral side, from the lumbar $3 / 4$ referred to the groin and no referred pain was obtained from the lumbar $2 / 3$, except for maximum pain and tenderness around the in jection site.

2) The above pain patterns turned out to be identical with that of pain obtained by osteovenography of the spine, irritation of interspinous ligaments by Kellgren and that of posterior primary division of spinal nerve by Ishida.

3) The pain distributed in the segmental pattern and never referred to the contralateral site of the injection.

4) The reflex spasm of the erector spinae was demonstrated in almost all cases and that of tensor faciae latae as well as hamstring was found in 8 patients out of 22 and the incidence seemed to increase by irritation of the lower lumbar spine. 
It was concluded that the referred pain from the facet articulation proved to play an important role in mechanism of the low back pain.

\title{
91. Stiffness of the Shoulder \\ Pathophysiological Aspect of View
}

\author{
Torai Iwahara, Kiyoshi Hirabayashi, Michitaka Kohno, \\ and Sadahisa HiJikata \\ Dept. of Orthopaedic Surg., School of Med., Keio University
}

Materials: Forty-six cases are examined, who came to our clinic with the chief complaint of neck pain or stiff shoulder suspecting of disc disorders. Twenty-four are female patients and twenty-two are male. The age of thirty-five cases are under thirty.

Method of study: Lateral X-ray films of maximal forward flexion and maximal backward extension and discogram are taken routinely. Seteroid hormone diluted with anaesthetic agent is injected into the disc spaces when discogram is taken. Evaluation of the improvement of the symptoms is made after the application of cervical traction and collar for a week period.

Result: Causative factors-Most of the female patients have engaged in knitting, type-writing, and dress-making on professional or non-professional basis. Past history revealed some cervical trauma in the most male patients. Both persistant flexed position of the cervical spine and trauma to the cervical spine are considered to be possible causative factors to disc disorders.

Abnormal localized kyphosis-Abnormal localized kyphosis are observed in seventy-four per cent of the cases, mostly at the level of disc between fifth and sixth cervical vertebrae, and secondly disc between fourth and fifth. Some cases have localized kyphosis at both levels. Many authors have reported same frequency of this finding in the cervical disc degeneration. Abnormal localized kyphosis extending from fourth and fith cervical vertebrae is frequently detected in the lateral X-ray of flexion, while that of between fifth and sixth can be found in the lateral $X$-ray of neutral position. In comparison with localized kyphosis and sites of complaints, the later can be explained as refered pain by sino-vertebral nerves. The apex disc of the abnormal localized kyphosis is most degenerated comparing to the upper or lower adjacent discs. This abnormal localized kyphosis itself is considered to represent the static functional disorder of cervical 